\title{
Design and Implementation of Novel LMI-Based Iterative Learning Robust Nonlinear Controller
}

\author{
Saleem Riaz $\left(\mathbb{D},{ }^{1}\right.$ Hui Lin $\left(\mathbb{D},{ }^{1}\right.$ Farkhanda Afzal $\left(\mathbb{D},{ }^{2}\right.$ and Ayesha Maqbool $\mathbb{D D}^{3}$ \\ ${ }^{1}$ School of Automation, Northwestern Polytechnical University, Shaanxi, Xi'an 170072, China \\ ${ }^{2}$ MCS, National University of Sciences and Technology, Islamabad 44000, Pakistan \\ ${ }^{3}$ Department of Computer Science, NUST Balochistan Campus (NBC), Quetta, Islamabad, Pakistan
}

Correspondence should be addressed to Hui Lin; linhui@nwpu.edu.cn

Received 31 January 2021; Revised 14 March 2021; Accepted 22 March 2021; Published 16 April 2021

Academic Editor: Dr Shahzad Sarfraz

Copyright (C) 2021 Saleem Riaz et al. This is an open access article distributed under the Creative Commons Attribution License, which permits unrestricted use, distribution, and reproduction in any medium, provided the original work is properly cited.

An iterative learning robust fault-tolerant control algorithm is proposed for a class of uncertain discrete systems with repeated action with nonlinear and actuator faults. First, by defining an actuator fault coefficient matrix, we convert the iterative learning control system into an equivalent unknown nonlinear repetitive process model. Then, based on the mixed Lyapunov function approach, we describe the stability of the nonlinear repetitive mechanism on time and trial indices and have appropriate conditions for the repeated control system's stability in terms of linear matrix inequality theory. Through LMI techniques, we have obtained satisfactory results and controller stability, and robustness against fault tolerance is also discussed in detail. Finally, the simulation results of the output tracking control of the two exemplary models verify the effectiveness of the proposed algorithm.

\section{Introduction}

Iterative learning control is suitable for controlled objects with repetitive motion (running) properties in a limited time interval. It uses the data generated during the previous iteration of the system to correct undesirable control signals and generate the control signals used in the current iteration to make the system control. The performance is gradually improved, and finally, the complete tracking in a limited time interval is achieved. In a comparison with other control methods, the iterative learning control method has a simple controller method, a small amount of calculation, and only less knowledge of dynamic characteristics and can get the precise control. The characteristics of complete tracking of the controlled object are as follows: this control technology is applied in industrial applications such as assembly line industrial robots and chemical intermittent processes. The process of the iterative learning control system can generally detect the timeline and batch on the tree of a two-dimensional repetitive process. Over the last few years, based on system 2D generation idea, learning control system analysis and design had tremendous attention. The study in [1] designed a PD-type iterative learning control algorithm for a linear continuous repetition system based on the $2 \mathrm{D}$ method and obtained the system with accurate tracking control; the authors of $[2,3]$ combined with the stability theory discrete process designed an iterative learning controller. The output error of the system converges monotonically, and the performance in the direction of the batch axis gradually improves with the repeated process. To optimize the replicated system's performance in both time and batch order, the study in [4] designed a good control architecture for the uncertain $2 \mathrm{D}$ system. It proposed a balanced optimization algorithm of robust iterative learning, which comprehended the repeated system's stable robustness in both directions [5].

Iterative learning control uses the tracking error and original data of the system to continuously modify the current input target, so that the system output can quickly track the expected output within a limited time [6-9]. Based on iterative learning control system that can be regarded as a kind of repeated processes based on batch axis and the timeline, the system control problem of the research in recent years came to the attention of academia at home and 
abroad and was successfully used in multiaxis truss type robot, injection molding machine, and electric motorcycle motor system in the practical repeat operations such as industrial object $[3,10-12]$. As the process of industrialization continues to accelerate, industrial systems are becoming more and more complex, and people's requirements for system reliability and safety are getting higher and higher, making fault diagnosis and fault-tolerant control in the past few decades [13-16]. Both academic and practical application fields have received more and more attention. Fault-tolerant control is divided into active fault-tolerant control and passive fault-tolerant control. In fact, most researches pay more attention to active fault-tolerant control. Fault estimation [17-20] is different from fault diagnosis; it can accurately estimate the magnitude and shape of the fault and thus is able to reconstruct the fault signal. Therefore, in existing literature, fault estimation is a prerequisite for fault-tolerant control and has achieved affluence of theoretical research results [21-23]. At present, the methods of fault estimation mainly include observer-based methods [24, 25], signal reconstruction-based methods $[26,27]$, and artificial intelligence-based methods $[28,29]$. The observer-based methods can accurately reflect the impact of faults on system performance and accuracy. The fault information has attracted the attention of domestic and foreign scholars in theoretical research. It mainly includes methods based on adaptive state observers [30-32], methods based on robust observers [33-36], and optimal $[9,37]$ and sliding mode observers approaches [38-41]. These methods have been continuously applied to network control systems [42-44], nonlinear systems [45], switching systems [46], fuzzy systems [47], parameter changing systems [48], and so on.

In control theory, the $\mathrm{RC}$ is a neutral-type delay, and the number is like endless poles. In 1988, "Scientific American" (English) mentioned that RCs could only stabilize unless there are zero degree relative plants [49]. Most technicians need to push the low-level filter on the delay control for a strictly proper plant. On this basis, the established system is improved to modify RCs (MRCs), and its low-level filter can reduce the steady state at the cost of high-frequency tracking signals [50]. That is to say, the stability and tracking functions in MRC have an intermediate point. Therefore, on the one hand, it is a stable system, and on the other hand, it is easily introduced into the distorted system when there is great uncertainty in the way [51-53].

However, it cannot be ignored that actuators in actual industrial systems with repetitive operation characteristics are often in high-frequency responding, and actuators are very prone to mechanical wear, failure, and other faults, which may not only reduce the control performance of the system but also affect the stability and safety of the system [7, 54-58]. B. Cichy and his team [59] discuss the design of robust iterative learning fault-tolerant control law for actuator fault linear systems and its convergence. The paper [60] further analyzes the robustness of the guaranteed iterative learning fault-tolerant control system and finally guarantees the reliability of the iterative learning repetition process on batch axis and time axis. However, the above-stated pieces of literature are all aimed at completely linear systems, while the real control systems inevitably have some nonlinear characteristics. In papers [61, 62], iterative learning control laws are designed for discrete repeated processes with nonlinear links, and stability analysis is carried out for these nonlinear systems by defining hybrid Lyapunov function and adaptive Lyapunov function based on batch axis and time axis, respectively. However, these methods do not fully consider the robustness of uncertainty in the systems, let alone discuss the fault shadow of nonlinear systems. Therefore, the design of robust iterative fault-tolerant control law for repetitive process systems with nonlinear links and uncertainties is an inevitable choice to improve the performance and reliability of system batches.

In this paper, the iterative learning in fault-tolerant control for a class of uncertain discrete nonlinear repetitive processes with unknown actuator faults is studied. By designing iterative learning fault-tolerant control law and defining the mixed Lyapunov function based on batch axis and time axis, the stability of the system under normal and failure conditions is discussed, respectively, and the sufficient conditions for the existence of robust fault-tolerant controller are given in the form of linear matrix inequality (LMI). Finally, this method is applied to the simulation of single-link manipulator and the injection velocity control process of molding process system to verify the effectiveness of the proposed method.

In this paper, for the convenience of description, the following assumptions are made for the matrix:

$\mathbf{X}, \mathbf{X}^{\mathrm{T}} \in R^{n}$ represents its transpose.

$\mathbf{X}<0$ represents a negative definite matrix

$\mathbf{X}>0$ denotes a positive definite matrix

* denotes the transpose of elements at the symmetric position of the matrix

$\|$.$\| represents the Euclidean norm$

$\forall$ any

\section{Problem Description}

Consider the following class of uncertain discrete time-invariant nonlinear systems running repeatedly:

$\left\{\begin{array}{l}x(t+1, k)=(A+\Delta A) x(t, k)+(B+\Delta B) u(t, k)+f(x(t, k)), \\ y(t, k)=(C+\Delta C) x(t, k)\end{array}\right.$

where $k=0,1, \ldots, N$ stands for batch, and the repeating time cycle within each batch is $0 \leq t \leq T$. $X(t, k) \in R^{n}$, $u(t, k) \in R^{l}$, and $y(t, k) \in R^{m}$ separately represent the state vector, input vector, and output vector of the system. Without loss of generality, suppose the initial boundary conditions of the system $x(0, k)=x 0$ and $u(t, 0)=u 0(t)$. Matrices $A, B$, and $C$ are, respectively, the corresponding dimension of the system matrix, and $\Delta A, \Delta B$, and $C \Delta$ are uncertainty [63] and satisfy the following relations: $\Delta A=H 1 \Xi F 1, \Delta B=H 1 \Xi F 2$, and $\Delta C=H 2 \Xi \mathrm{F} 2$, including $H 1, H 2, F 1$, and $F 2$ to already know the certainty of the 
matrix; $\Xi \Xi T \leq$ I or less bounded constraint condition where I is the identity matrix. In addition, $f(x(t, k)) \in R^{n}$ represents a nonlinear vector function obtained from the modeling of the system's nonlinear link, assuming that the following conditions are met:

$$
\|f(x(t, k+1))-f(x(t, k))\| \leq \zeta\|x(t, k+1)-x(t, k)\| .
$$

Here, $\zeta>0$ is Lipschitz constant. For the vector element $\mathrm{UI}(t, k)$ that is input to the system, $u_{i}^{F}(t, k)$ represents the output when an actuator fails in the system. Actuator fault model is further defined:

$$
u_{i}^{F}(t, k)=\alpha_{i} u_{i}(t, k), \quad i=1,2, \ldots, l,
$$

where the actuator fault coefficient $\alpha_{i}$ is unknown but meets the following conditions:

$$
0 \leq \underline{\alpha}_{t} \leq \alpha_{i} \leq \bar{\alpha}_{i}, \quad i=1,2, \ldots, l,
$$

where the properties $\underline{\alpha}_{i}\left(\underline{\alpha}_{i} \leq 1\right)$ and $\bar{\alpha}_{i}\left(\bar{\alpha}_{i} \geq 1\right)$ are known as scalars.

If $\underline{\alpha}_{i}=\bar{\alpha}_{i}=1$, it means that the control system actuator is working normally; that is, $\mathbf{u}_{i}^{\mathrm{F}}(t, k)=\mathbf{u}_{i}(t, k)$. If $\alpha_{i}=0$, it represents the complete failure of the actuator due to the damage caused by various emergencies; $\alpha_{i}>0$ represents a partial actuator failure due to normal conditions such as

$$
\begin{aligned}
\mathbf{u}^{\mathrm{F}} & =\left[u_{1}^{\mathrm{F}}, u_{2}^{\mathrm{F}}, \ldots, u_{l}^{\mathrm{F}}\right]^{\mathrm{T}}, \\
\overline{\boldsymbol{\alpha}} & =\operatorname{diag}\left\{\bar{\alpha}_{1}, \bar{\alpha}_{2}, \ldots, \bar{\alpha}_{l}\right\}, \\
\underline{\boldsymbol{\alpha}} & =\operatorname{diag}\left\{\underline{\alpha}_{1}, \underline{\alpha}_{2}, \ldots, \underline{\alpha}_{l}\right\}, \\
\boldsymbol{\alpha} & =\operatorname{diag}\left\{\alpha_{1}, \alpha_{2}, \ldots, \alpha_{l}\right\} .
\end{aligned}
$$

On this arrangement, $\beta=\operatorname{diag}\left\{\beta_{1}, \beta_{2}, \ldots, \beta_{l}\right\}, \beta_{0}=\operatorname{diag}$ $\left\{\beta_{10}, \beta_{20}, \ldots, \beta_{00}\right\}, \quad \beta_{i}=\left(\left(\bar{\alpha}_{i}+\alpha_{i}\right) / 2\right)$, and $\beta_{i 0}=\left(\left(\bar{\alpha}_{i}-\alpha_{i}\right) /\right.$ $\left.\left(\bar{\alpha}_{i}+\alpha_{i}\right)\right), i=1,2, \ldots, l$. There must be some unknown matrices $\alpha_{0}$ :

$$
\begin{gathered}
\alpha=\left(I+\alpha_{0}\right) \beta, \\
I \boldsymbol{\alpha}_{0} \mid \leq \boldsymbol{\beta}_{0} \leq \mathbf{I} .
\end{gathered}
$$

Other $\alpha_{0}=\operatorname{diag}\left\{\alpha_{10}, \alpha_{20}, \ldots, \alpha_{l 0}\right\} \quad$ and $\left|\alpha_{0}\right|=\operatorname{diag}$ $\left\{\left|\alpha_{10}\right|,\left|\alpha_{20}\right|, \ldots,\left|\alpha_{l 0}\right|\right\}$.

Therefore, discrete time-invariant nonlinear system (1) with actuator fault can be expressed as

$$
\left\{\begin{array}{l}
x(t+1, k)=(A+\Delta A) x(t, k)+(B+\Delta B) \omega t(t, k)+f(x(t, k)), \\
y(t, k)=(C+\Delta C) x(t, k) .
\end{array}\right.
$$

Uncertain discrete nonlinear steady-state systems contain actuator failures (7), and the control goal of this paper is to meet the condition (6) in the case of unknown actuator failures. Based on the iterative learning, fault-tolerant control input makes the system output so that the control system can gradually track the output after a certain required number of iterations, i.e,

$$
\sup _{0 \leq i \leq T}\left(y_{x}(t)-y(t, k) \mid<\varepsilon\right.
$$

\section{Iterative Learning Control Law Design}

According to the control objective of this paper, the output tracking error $e(t, k)$ is defined as follows:

$$
e(t, k)=y_{k}(t)-y(t, k) \text {. }
$$

For system (8), the following control law is designed:

$$
u(t, k+1)=u(t, k)+\Delta u(t, k+1),
$$

where $u(t, k+1)$ is the system control input of the current batch; $u(t, k)$ is the control input of the previous batch; $\Delta u(t, k+1)$ is to modify control system input amount of updates.

For the convenience of analysis, define

$$
\left\{\begin{array}{l}
\eta(t+1, k+1)=x(t, k+1)-x(t, k), \\
\varphi(t, k+1)=f(x(t-1, k+1))-f(x(t-1, k)) .
\end{array}\right.
$$

Therefore, the modified update quantity in iterative learning control law (11) can be further obtained as

$$
\Delta u(t, k+1)=K_{1} \eta(t+1, k+1)+K_{2} e(t+1, k),
$$

where $K_{1}$ and $K_{2}$ are unknown undetermined matrices.

By substituting the iterative learning fault-tolerant control law form (11) into the nonlinear system (8), the state-space model of discrete repeated processes can be obtained in the following form:

$$
\left\{\begin{array}{l}
\boldsymbol{\eta}(t+1, k+1)=\widehat{\mathbf{A}} \boldsymbol{\eta}(t, \mathbf{k}+1)+\widehat{\mathbf{B}} \mathbf{e}(t, \mathbf{k})+\boldsymbol{\varphi}(t, \mathbf{k}+1) \\
\mathbf{e}(t, \mathbf{k}+1)=\widehat{\mathbf{C}}_{\boldsymbol{\eta}}(t, \mathbf{k}+1)+\widehat{\mathbf{D}} e(t, \mathbf{k})+\widehat{\mathbf{k}}_{\varphi}(t, \mathbf{k}+1)
\end{array}\right.
$$

Here,

$$
\begin{aligned}
& \widehat{A}=(A+\Delta A)+(B+\Delta B) \alpha K_{1}, \\
& \widehat{B}=(B+\Delta B) \alpha K_{2}, \\
& \widehat{C}=-(C+\Delta C)\left((A+\Delta A)+(B+\Delta B) \alpha K_{1}\right), \\
& \widehat{D}=I-(C+\Delta C)(B+\Delta B) \alpha K_{2}, \\
& \widehat{E}=-(C+\Delta C) .
\end{aligned}
$$

Obviously, discrete repetitive process model (14) is a nonlinear repeated process, including $\eta(t+1, k+1)$ and $\mathbf{e}(t, \mathbf{k})$, respectively, and represents the time and batch variable on the axis direction, $\varphi(t, \mathbf{k}+1)$ is to enter the present process of iterative nonlinear input item, and conventional stability analysis of linear systems of KYP lemma methods cannot be directly used to solve the repetitive process system with nonlinear term.

\section{Stability Analysis}

The following lemma is given in advance for subsequent stability analysis.

Lemma 1 (see [64]). If $\Omega_{1}$ and $\Omega_{2}$ are, respectively, about variable $\omega \in R^{n}$ of two dimensions of the same symmetric matrix, then it shows the following equation: 
$\vec{\omega}^{T} \Omega_{1} \omega \leq 0, \forall \omega \neq 0, \omega^{T} \Omega_{2}, \omega \leq 0$. Founded, there is always the $\exists \tau \geq 0$ can make $\Omega_{1}-\tau \Omega_{2}<0$.

Lemma 2 (see [65]). The matrix $\Phi$ with a given appropriate dimension is equal to $\Phi=\Phi^{T}$. And then for any matrix $\Delta^{T} \Delta \leq I$, or $\Delta$ less inequality below $\Phi+\mathbf{X} \Delta \mathbf{Y}+\mathbf{Y}^{\mathrm{T}} \Delta^{\mathrm{T}} \mathbf{X}^{\mathrm{T}}<0$. If and only if there is $\varepsilon>0$, such that $\Phi+\varepsilon \mathbf{X X}^{\mathrm{T}}+\varepsilon^{-1} \mathbf{Y}^{\mathrm{T}} \mathbf{Y}<0$.

Lemma 3 (see [66]). For matrices of given dimensions $\Gamma, \Lambda$, and $\Sigma$, existing matrix $W$ makes linear matrix inequality (LMI), there exists a matrix $\mathrm{W}$. This makes the following linear matrix inequality true: $\Gamma+\operatorname{sym}\left\{\Lambda^{\mathrm{T}} \mathbf{W} \Sigma\right\}<0$, if and only if the following two inequalities about the matrix $W$ are true $\Lambda^{\perp \mathrm{T}} \Gamma \Lambda^{\perp}<0_{\mathbf{r}} \Sigma^{\perp \mathrm{T}} \Gamma \Sigma^{\perp}<0$.

Theorem 1. If the existence of the matrix $P>0$ makes the following LMI true, then the nonlinear discrete repeat process (14) is stable.

$$
\left[\begin{array}{ccc}
\widehat{A}^{\mathrm{T}} \mathbf{P} \widehat{\mathbf{A}}-\mathbf{P}+\widehat{\mathbf{C}}^{\mathrm{T}} \widehat{\mathbf{C}} & \widehat{\mathbf{A}}^{\mathrm{T}} \mathbf{P} \widehat{\mathbf{B}}+\widehat{\mathbf{C}}^{\mathrm{T}} \widehat{\mathbf{D}} & \widehat{\mathbf{A}}^{\mathrm{T}} \mathbf{P}+\widehat{\mathbf{C}}^{\mathrm{T}} \widehat{\mathbf{E}} \\
\widehat{\mathbf{B}}^{\mathrm{T}} \mathbf{P} \widehat{\mathbf{A}}+\widehat{\mathbf{D}}^{\mathrm{T}} \mathbf{C} & \widehat{\mathbf{B}}^{\mathrm{T}} \mathbf{P} \widehat{\mathbf{B}}+\widehat{\mathbf{D}}^{\mathrm{T}} \widehat{\mathbf{D}}-\mathbf{I} & \widehat{\mathbf{B}}^{\mathrm{T}} \mathbf{P}+\widehat{\mathbf{D}}^{\mathrm{T}} \widehat{\mathbf{E}} \\
\mathbf{P} \widehat{\mathbf{A}}+\widehat{\mathbf{E}}^{\mathrm{T}} \widehat{\mathbf{C}} & \mathbf{P} \widehat{\mathbf{B}}+\widehat{\mathbf{E}}^{\mathrm{T}} \widehat{\mathbf{D}} & \mathbf{P}+\widehat{\mathbf{E}}^{\mathrm{T}} \widehat{\mathbf{E}}
\end{array}\right]<0 .
$$

It is proved that the mixed Lyapunov function for the discrete nonlinear repeat process (14) is constructed in the following form with respect to the time axis and batch axis:

$$
\begin{aligned}
\mathbf{V}(t, k)= & \mathbf{V}_{1}(t, k)+\mathbf{V}_{2}(t, k)=\mathbf{\eta}^{T}(t, k+1) \mathbf{P}_{\boldsymbol{\eta}}(t, k+1) \\
& +\mathbf{e}^{T}(t, \mathbf{k}) \mathbf{R} \boldsymbol{e}(t, \mathbf{k}),
\end{aligned}
$$

and define

$$
\begin{aligned}
\Delta \mathbf{V}_{1}(t, k)= & \mathbf{\eta}^{T}(t+1, k+1) \mathbf{P} \eta(t+1, k+1) \\
& -\boldsymbol{\eta}^{T}(t, k+1) \mathbf{P}_{\boldsymbol{\eta}}(t, k+1), \\
\Delta \mathbf{V}_{2}(t, k)= & \mathbf{e}^{T}(t, k+1) \mathbf{R} e(t, k+1)-\mathbf{e}^{T}(t, k) \mathbf{R} e(t, k), \\
\Delta V(t, k)= & \Delta V_{1}(t, k)+\Delta V_{2}(t, k),
\end{aligned}
$$

where $P>0, R=I \quad b>0$. According to stability theorem, when $\Delta V(t, k)<0$, discrete nonlinear repeat procedure (17) is stable: namely,

$$
\Delta V_{1}(t, k)+\Delta V_{2}(t, k)<0 .
$$

Plug in $\eta(t+1, k+1)$ and $\mathrm{e}(t, k+1)$ into equation (19), and get

$$
\begin{aligned}
& {\left[\widehat{\mathbf{A}}_{\boldsymbol{\eta}}(t, \mathbf{k}+1)+\widehat{\mathbf{B}} e(t, \mathbf{k})+\varphi(t, \mathbf{k}+1)\right]^{\top} \mathbf{P},} \\
& {\left[\widehat{\mathbf{A}}_{\boldsymbol{\eta}}(t, \mathbf{k}+1)+\widehat{\mathbf{B}} e(t, \mathbf{k})+\varphi(t, \mathbf{k}+1)-\eta(t, \mathbf{k}+1)\right]^{\top} \mathbf{P},} \\
& \boldsymbol{\eta}(t, k+1)+\widehat{\mathbf{C}} \boldsymbol{\eta}(t, k+1)+\widehat{\mathbf{D}} e(t, \mathbf{k})+\widehat{\mathbf{E}} \varphi(t, k+1)^{\top}, \\
& \widehat{\mathbf{C}} \boldsymbol{\eta}(t, k+1)+\widehat{\mathbf{D}} e(t, \mathbf{k})+\widehat{\mathbf{E}} \varphi(t, k+1)^{\top}-e(t, k)^{\top} e(t, k)<0 .
\end{aligned}
$$

After transformation, the following inequalities can be obtained:

$$
\mathbf{X}^{\mathrm{T}}\left[\begin{array}{ccc}
\widehat{\mathbf{A}}^{\mathrm{T}} \mathbf{P} \widehat{\mathbf{A}}+\mathbf{P}+\widehat{\mathbf{C}}^{\mathrm{T}} \widehat{\mathbf{C}} & \widehat{\mathbf{A}}^{\mathrm{T}} \mathbf{P} \widehat{\mathbf{B}}+\widehat{\mathbf{C}}^{\mathrm{T}} \widehat{\mathbf{D}} & \widehat{\mathbf{A}}^{\mathrm{T}} \mathbf{P}+\widehat{\mathbf{C}}^{\mathrm{T}} \widehat{\mathbf{E}} \\
\widehat{\mathbf{B}}^{\mathrm{T}} \mathbf{P} \widehat{\mathbf{A}}+\widehat{\mathbf{D}}^{\mathrm{T}} \widehat{\mathbf{C}} & \widehat{\mathbf{B}}^{\mathrm{T}} \mathbf{P} \widehat{\mathbf{B}}+\widehat{\mathbf{D}}^{\mathrm{T}} \widehat{\mathbf{D}}-\mathbf{I} \widehat{\mathbf{B}}^{\mathrm{T}} \mathbf{P}+\widehat{\mathbf{D}}^{\mathrm{T}} \widehat{\mathbf{E}} \widehat{\mathbf{E}} \\
\mathbf{P} \widehat{\mathbf{A}}+\widehat{\mathbf{E}}^{\mathrm{T}} \widehat{\mathbf{C}} & \mathbf{P} \widehat{\mathbf{B}}+\widehat{\mathbf{E}}^{\mathrm{T}} \widehat{\mathbf{D}} & \mathbf{P}+\widehat{\mathbf{E}}^{\mathrm{E}} \widehat{\mathbf{E}}
\end{array}\right]
$$

Here, $X=\left[\begin{array}{c}\eta(t, k+1) \\ e(t, k) \\ \varphi(t, k+1)\end{array}\right]$, thus directly obtaining the conclusion of Theorem 1 .

There is coupling between unknown matrix variables in the conclusion of Theorem 1, so K1 and $\mathrm{K} 2$ of iterative learning fault-tolerant control correction and update quantity (13) cannot be calculated by direct application of the LMI tool. Therefore, based on the conclusion of Theorem 1 , it is further discussed and Theorem 2 is obtained.

Theorem 2. Regardless of system (1), the uncertainty, i.e., $\Delta A$, $\Delta \Delta C$, and $B$ are assumed to 0 , if there is $A$ positive definite matrix $Q>0$, matrix $N 1$, and $N 2$ and scalar tau $>0$, epsilon $1>0$ make the matrix inequality $\Omega<0$, the nonzero symmetric matrix elements: $\Omega 11=044=-Q, \quad \Omega 14=(A Q+B \beta N 1) T$, $\Omega 15=[-C(A Q+B \beta N 1) T, \Omega 16=Q, \Omega 18=(\beta N 1) T, \Omega 22=-\tau I$, $\Omega 24=(B \beta N 2) T, \quad \Omega 25=(\tau I-C B \beta N 2) T, \quad \Omega 28=(\beta N 2) T$, $\Omega 33=-I, \Omega 34=I, \Omega 35=(-C) T, \Omega 47=\varepsilon 1 \beta 0 B, \quad \Omega 55=-\tau I$, $\Omega 57=-\varepsilon 1 \beta 0 C B, \Omega 66=-\rho I, \Omega 77=088=-\varepsilon 11$, then the nonlinear discrete repetition process (14) is stable under the action of the actuator fault satisfies the admissible condition (7) and the iterative learning fault-tolerant control law (11), where $\rho=\zeta^{-2}$, modify the gain matrix of update quantity (13) $K_{1}=N_{1} Q^{-1}, K_{2}=\tau^{-1} N_{2}$. The proof notes that the following equivalent conditions can be obtained according to equation (2):

$$
\begin{gathered}
{\left[f(x(t-1, k+1))-f(x(t-1, k))^{T}\right]} \\
{[f(x(t-1, k+1))-f(x(t-1, k))]} \\
\leq \zeta^{2}[x(t-1, k+1)-x(t-1, k] \\
\quad \cdot[x(t-1, k+1)-x(t-1, k)]] .
\end{gathered}
$$

Substituting equation (12) into the above equation, we can obtain $\varphi(t, k+1) T \varphi(t, k+1) \leq \zeta 2 \eta(t, k+1) T \eta(t, k+1)$.

That is,

$$
\mathbf{X}^{T} \boldsymbol{\Omega}_{2} \mathbf{X} \leq 0
$$

and it can be known from Theorem 1 that $\mathbf{X}^{T} \Omega_{1} \mathbf{X} \leq 0$, in which

$$
\begin{aligned}
\boldsymbol{\Omega}_{1} & =\left[\begin{array}{ccc}
\widehat{\mathbf{A}}^{\mathrm{T}} \mathbf{P} \widehat{\mathbf{A}}-\mathbf{P}+\widehat{\mathbf{C}}^{\mathrm{T}} \widehat{\mathbf{C}} & \widehat{\mathbf{A}}^{\mathrm{T}} \mathbf{P} \widehat{\mathbf{B}}+\widehat{\mathbf{C}}^{\mathrm{T}} \widehat{\mathbf{D}} & \widehat{\mathbf{A}}^{\mathrm{T}} \mathbf{P}+\widehat{\mathbf{C}}^{\mathrm{T}} \widehat{\mathbf{E}} \\
\widehat{\mathbf{B}}^{\mathrm{T}} \mathbf{P} \widehat{\mathbf{A}}+\widehat{\mathbf{D}}^{\mathrm{T}} \widehat{\mathbf{C}} & \widehat{\mathbf{B}}^{\mathrm{T}} \mathbf{P} \widehat{\mathbf{B}}+\widehat{\mathbf{D}}^{\mathrm{T}} \widehat{\mathbf{D}}-\mathbf{I} & \widehat{\mathbf{B}}^{\mathrm{T}} \mathbf{P}+\widehat{\mathbf{D}}^{\mathrm{T}} \widehat{\mathbf{E}} \\
\mathbf{P} \widehat{\mathbf{A}}+\widehat{\mathbf{E}}^{\mathrm{T}} \widehat{\mathbf{C}} & \mathbf{P} \widehat{\mathbf{B}}+\widehat{\mathbf{E}}^{\mathrm{T}} \widehat{\mathbf{D}} & \mathbf{P}+\widehat{\mathbf{E}}^{\mathrm{T}} \widehat{\mathbf{E}}
\end{array}\right], \\
\boldsymbol{\Omega}_{2} & =\left[\begin{array}{ccc}
-\zeta^{2} & 0 & 0 \\
0 & 0 & 0 \\
0 & 0 & I
\end{array}\right] .
\end{aligned}
$$


Then, the following inequality can be obtained through Lemma 1:

$$
\mathbf{\Omega}_{1}-\tau \mathbf{\Omega}_{2}<0,
$$

and $\tau>0$ type can be rewritten as $\tau-1$ on $\Omega 1-\Omega 2<0$; namely,

$$
\boldsymbol{\theta}=\left[\begin{array}{ccc}
\boldsymbol{\theta}_{11} & \boldsymbol{\theta}_{12} & \boldsymbol{\theta}_{13} \\
* & \boldsymbol{\theta}_{22} & \boldsymbol{\theta}_{23} \\
* & * & \boldsymbol{\theta}_{33}
\end{array}\right]<0,
$$

where symmetric elements are

$$
\begin{aligned}
\boldsymbol{\Theta}_{11} & =\tau^{-1} \widehat{\mathbf{A}}^{\mathrm{T}} \mathbf{P} \widehat{\mathbf{A}}-\tau^{-1} \mathbf{P}+\tau^{-1} \widehat{\mathbf{C}}^{\top} \widehat{\mathbf{C}}+\zeta^{2} \mathbf{I}, \\
\boldsymbol{\theta}_{12} & =\tau^{-1} \widehat{\mathbf{A}}^{\top} \mathbf{P} \widehat{\mathbf{B}}+\tau^{-1} \widehat{\mathbf{C}}^{*} \mathbf{D}, \boldsymbol{\theta}_{13}=\tau^{-1} \widehat{\mathbf{A}}^{\top} \mathbf{P}+\tau^{-1} \widehat{\mathbf{C}}^{\mathrm{T}} \mathbf{E}, \\
\boldsymbol{\theta}_{n} & =\tau^{-1} \widehat{A}^{\top} \mathbf{P}+\tau^{-1} \widehat{\mathbf{C}}^{\top} \mathbf{E}, \boldsymbol{\theta}_{2}=\tau^{-1} \widehat{\mathbf{B}}^{\top} \mathbf{P}+\tau^{-1} \mathbf{D}^{\top} \mathbf{E}, \boldsymbol{\theta}_{33} \\
& =\tau^{-1} \mathbf{P}+\tau^{-1} \mathbf{E}^{\top} \mathbf{E}-\mathbf{I} .
\end{aligned}
$$

By applying the Schur complement lemma to equation (26), the following inequality is obtained:

$$
\left[\begin{array}{ccccc}
-\tau^{-1} \mathbf{P}+\zeta^{2} \mathbf{I} & 0 & 0 & \widehat{\mathbf{A}}^{\mathrm{T}} & \widehat{C}^{\mathrm{T}} \\
* & -\tau^{-1} \mathbf{I} & 0 & \widehat{B}^{\mathrm{T}} & \widehat{D}^{\mathrm{T}} \\
\star & \star & -\mathbf{I} & I & \widehat{E}^{\mathrm{T}} \\
\star & \star & \star & -\tau \mathbf{P}^{-1} & 0 \\
\star & \star & \star & \star & -\tau \mathbf{I}
\end{array}\right]<0 .
$$

Lemma, the following results can be obtained:

$$
\left[\begin{array}{cccccc}
-Q^{-1} & 0 & 0 & \widehat{A}^{\mathrm{T}} & \widehat{C}^{\mathrm{T}} & I \\
* & -\tau^{-1} I & 0 & \widehat{B}^{\mathrm{T}} & \widehat{D}^{\mathrm{T}} & 0 \\
\star & * & -I & I & \widehat{E}^{\mathrm{T}} & 0 \\
\star & \star & \star & -\mathrm{Q} & 0 & 0 \\
\star & \star & \star & \star & -\tau I & 0 \\
\star & \star & \star & \star & \star & -\rho I
\end{array}\right]<0 .
$$

Further multiply (29) on both sides by $\operatorname{diag}\{\mathbf{Q}, \tau \mathbf{I}, \mathbf{I}, \mathbf{I}, \mathbf{I}, \mathbf{I}\}$ and its transpose, and let $N_{1}=K_{1} Q, N_{2}=\tau K_{2} ; \quad$ then $\mathbf{A}+\mathbf{B} \alpha \mathbf{K}_{1}, \widehat{B}=\mathbf{B} \alpha \mathbf{K}_{2}$, $\widehat{\mathbf{C}}=-\mathbf{C}\left(\mathbf{A}+\mathbf{B} \alpha \mathbf{K}_{1}\right), \widehat{\mathbf{D}}=\mathbf{I}-\mathbf{C B} \alpha \mathbf{K}_{2}, \widehat{\mathbf{E}}=-\mathbf{C}$. If we put in this equation, we will get

$$
\left[\begin{array}{cccccc}
-Q & 0 & 0 & \left(A Q+B \alpha N_{1}\right)^{\mathrm{T}} & {\left[-C\left(A Q+B \alpha N_{1}\right)\right]} & \mathrm{Q} \\
* & -T I & 0 & \left(B \alpha N_{2}\right)^{\mathrm{T}} & \left(\tau I-C B \alpha N_{2}\right)^{\mathrm{T}} & 0 \\
\star & \star & -I & I & (-C)^{\mathrm{T}} & 0 \\
\star & \star & \star & -Q & 0 & 0 \\
\star & \star & \star & \star & -\tau I & 0 \\
\star & \star & \star & \star & \star & -\rho I
\end{array}\right]<0,
$$

and then we obtain $\alpha=\left(\mathbf{I}+\alpha_{0}\right) \beta$ into this equation and get

Here,

$$
Z+X \bar{E} Y+Y^{T} \bar{E} X^{T}<0 .
$$

$$
\begin{aligned}
& Z=\left[\begin{array}{cccccc}
-Q & 0 & 0 & \left(A Q+B \beta N_{1}\right)^{\mathrm{T}} & {\left[-C\left(A Q+B \beta N_{1}\right)\right]} & Q \\
* & -\tau I & 0 & \left(B \beta N_{2}\right)^{\mathrm{T}} & \left(\tau I-C B \beta N_{2}\right)^{\mathrm{T}} & 0 \\
\star & \star & -I & I & (-C)^{\mathrm{T}} & 0 \\
\star & \star & \star & -Q & 0 & 0 \\
\star & \star & \star & \star & -\tau I & 0 \\
\star & \star & \star & \star & \star & -\rho I
\end{array}\right] \text {, } \\
& X=\left[\begin{array}{lllllll}
0 & 0 & 0 & \mathbf{B}^{\mathrm{T}} & (-\mathbf{C B})^{\mathrm{T}} & 0
\end{array}\right]^{\mathrm{T}}, \\
& \bar{\Xi}=\boldsymbol{\alpha}_{0}, \\
& \mathbf{Y}=\left[\begin{array}{lllllll}
\boldsymbol{\beta} \mathbf{N}_{1} & \boldsymbol{\beta} N_{2} & 0 & 0 & 0 & 0
\end{array}\right] .
\end{aligned}
$$


According to Lemma 2, it is further obtained that

$$
\begin{aligned}
& Z+\varepsilon_{1} \boldsymbol{\beta}_{0}^{2} \mathbf{X X}^{\top}+\varepsilon_{1}^{-1} \mathbf{Y}^{\top} \mathbf{Y}<0 \\
& Z+\left[\begin{array}{lll}
\varepsilon_{1}^{(1 / 2)} \boldsymbol{\beta}_{0} \mathbf{X} & \varepsilon_{1}^{-(1 / 2)} \mathbf{Y}^{\top}
\end{array}\right]\left[\begin{array}{c}
\varepsilon_{1}^{(1 / 2)} \boldsymbol{\beta}_{0} \mathbf{X}^{\top} \\
\varepsilon_{1}^{-(1 / 2)} \mathbf{Y}
\end{array}\right]<0 .
\end{aligned}
$$

Using Schur complement lemma again, equation (34) can be written directly as

$$
\left(\begin{array}{cc}
Z & \varepsilon_{1}^{Z} \boldsymbol{\beta}_{0} \mathbf{X} \varepsilon_{1}^{-(1 / 2)} \mathbf{Y}^{\mathrm{T}} \\
* & -I
\end{array}\right)<0
$$

Multiplying (35) on both sides by $\operatorname{diag}\left\{\mathbf{I}, \mathbf{I}, \varepsilon_{1}^{(1 / 2)} \mathbf{I}, \varepsilon_{1}^{(1 / 2)} \mathbf{I}\right\}$ and its transpose, we get $\Omega<0$.

On the basis of Theorem 2, we further consider the stability of discrete nonlinear repetitive process (14) with uncertainty and design of iterative learning robust faulttolerant controller. Since Lemma 2 cannot be directly used in the proof process after considering uncertainty, we need to make the following equivalent transformation for Theorem 2. First, we define it as follows:

$$
\begin{aligned}
& \mathbf{R}_{1}=\left[\left(\mathbf{A Q}+\mathbf{B} \boldsymbol{\beta} \mathbf{N}_{1}\right) \quad \mathbf{B} \boldsymbol{\beta} \mathbf{N}_{2} \begin{array}{lllllll}
0 & 0 & 0 & 0 & 0 & 0
\end{array}\right]^{\mathrm{T}} \text {, } \\
& J_{1}=\left[\begin{array}{llllllll}
0 & 0 & 0 & 0 & -C^{\mathrm{T}} & 0 & 0 & 0
\end{array}\right]^{\mathrm{T}} \text {, } \\
& \text { - }\left[\begin{array}{ll}
I & J_{1}
\end{array}\right]\left[\begin{array}{cc}
\boldsymbol{\Phi}_{1} & \mathbf{R}_{1} \\
\mathbf{R}_{1}^{\mathrm{T}} & 0
\end{array}\right]\left[\begin{array}{c}
\mathbf{I} \\
\mathbf{J}_{1}^{\mathrm{T}}
\end{array}\right]<0 .
\end{aligned}
$$

If the matrix $\Gamma=\left[\begin{array}{cc}\Phi_{1} & \mathbf{R}_{1} \\ \mathbf{R}_{1}^{\dagger} & 0\end{array}\right], W=\left[\begin{array}{l}W_{1} \\ W_{2}\end{array}\right]$, and $\Lambda=\mathbf{I}$, $\Sigma=\left[J_{1}^{\mathrm{T}}-I\right]$, using Lemma 3, the following inequality can be obtained:

$$
\left[\begin{array}{cc}
\boldsymbol{\Phi}_{1} & \mathbf{R}_{1} \\
\mathbf{R}_{1}^{\mathrm{T}} & 0
\end{array}\right]+\operatorname{sym}\left\{\left[\begin{array}{c}
\mathbf{W}_{1} \\
\mathbf{W}_{2}
\end{array}\right]\left[\begin{array}{ll}
\mathbf{J}_{1}^{\mathrm{T}} & -\mathrm{I}
\end{array}\right]\right\}<0 .
$$

Here,

$$
\mathbf{W}_{1}=\left[\begin{array}{llllllll}
\mathbf{W}_{11}^{T} & \mathbf{W}_{12}^{T} & \mathbf{W}_{13}^{T} & \mathbf{W}_{14}^{T} & \mathbf{W}_{15}^{T} & \mathbf{W}_{16}^{T} & \mathbf{W}_{17}^{T} & \mathbf{W}_{18}^{T}
\end{array}\right]^{T} .
$$

Similarly, the above formula is sorted out in the same way again as follows:

$$
\begin{aligned}
\mathbf{R}_{2} & =\left[\begin{array}{lllllllll}
0 & 0 & 0 & 0 & \left(-\boldsymbol{\varepsilon}_{1} \boldsymbol{\beta}_{0} \mathbf{C}\right)^{\mathrm{T}} & 0 & 0 & 0 & 0
\end{array}\right]^{\mathrm{T}}, \\
\mathbf{J}_{2} & =\left[\begin{array}{lllllllll}
0 & 0 & 0 & 0 & 0 & 0 & \mathbf{B} & 0 & 0
\end{array}\right]^{\mathrm{T}},
\end{aligned}
$$

and make $\Gamma=\left[\begin{array}{cc}\Phi_{2} & \mathbf{R}_{2} \\ \mathbf{R}_{2}^{\mathrm{T}} & 0\end{array}\right], W=\left[\begin{array}{l}W_{2} \\ W_{3}\end{array}\right]$, and $\Sigma=\left[\begin{array}{cc}J_{2}^{\mathrm{T}} & -I\end{array}\right]$.

Theorem 3. Considers the case where system (1) has uncertainty, where it is false

$$
\boldsymbol{\Psi}=\left[\begin{array}{cc}
\boldsymbol{\Phi}_{2} & \mathbf{R}_{2} \\
\mathbf{R}_{2}^{\mathrm{T}} & 0
\end{array}\right]+\operatorname{sym}\left\{\left[\begin{array}{c}
\mathbf{W}_{2} \\
\mathbf{W}_{3}
\end{array}\right]\left[\begin{array}{ll}
\mathbf{J}_{2}^{\mathrm{T}} & -\mathbf{I}
\end{array}\right]\right\}<0 .
$$

In which, $W_{3}=\left[\begin{array}{llllll}W_{31}^{T} & W_{32}^{T} & \ldots & \ldots & W_{39}^{T} & W_{4}^{T} T\end{array}\right]$ Theorem 3 (1) the uncertainty of the system, including false set is $\Delta A=H 1 \Xi F 1, \Delta B=H 1 \Xi F 2, \Delta C=H 2 \Xi F 2$, and if there is a positive definite matrix $\mathrm{Q}>0$, matrix $N 1, N 2$ and scalar tau $>0, \Lambda>0, \varepsilon 1>0$ make matrix inequality $\Upsilon<0$ as follows:

$$
\begin{aligned}
\mathbf{Y} & =\left[\begin{array}{cc}
\mathbf{Y}_{11} & \mathbf{Y}_{12} \\
* & \mathbf{Y}_{22}
\end{array}\right]<0, \\
\mathbf{Y}_{11} & =\left[\begin{array}{cc}
\boldsymbol{\Psi}_{11} & \boldsymbol{\Psi}_{12} \\
* & \boldsymbol{\Psi}_{22}
\end{array}\right], \\
\mathbf{Y}_{12} & =\left[\begin{array}{ll}
\boldsymbol{\Psi}_{31} & \boldsymbol{\Psi}_{32}
\end{array}\right], \\
\mathbf{Y}_{22} & =\operatorname{diag}\{-\lambda \mathbf{I}-\lambda \mathbf{I}-\lambda \mathbf{I}-\lambda \mathbf{I}-\lambda \mathbf{I}-\lambda \mathbf{I}\} .
\end{aligned}
$$

The discrete repetition process (14) was stable under the condition of failure tolerance (7) and iterative learning robust fault-tolerant control law (11), and the modified update quantity (13) was the gain matrix $\mathbf{K}_{1}=\mathbf{N}_{1} \mathbf{Q}^{-1}$, $\mathbf{K}_{2}=\tau^{-1} \mathbf{N}_{2}$.

The proof of formula (40) can be transformed into, when the system uncertainty is further considered. $\mathbf{Y}_{1}+\mathbf{M} \Xi \mathbf{N}+$ $\mathbf{N}^{T} \Xi \mathbf{M}^{T}<0$

Here,

$$
\begin{aligned}
\mathbf{Y}_{1} & =\left[\begin{array}{cc}
\mathbf{Y}_{11} & \mathbf{Y}_{12} \\
* & \mathbf{Y}_{22}
\end{array}\right], \\
\mathbf{M} & =\left[\begin{array}{llllllllll}
0 & 0 & 0 & \mathbf{H}_{1}^{\mathrm{T}} & 0 & 0 & 0 & 0 & \mathbf{H}_{1}^{\mathrm{T}} & 0 \\
0 & 0 & 0 & 0 & \mathbf{H}_{2}^{\mathrm{T}} & 0 & 0 & 0 & 0 & 0 \\
0 & 0 & 0 & 0 & 0 & 0 & \mathbf{F}_{2} & 0 & 0 & 0
\end{array}\right], \\
N & =\Psi_{32}^{\mathrm{T}} .
\end{aligned}
$$

Using Lemma 2 and conditions $\Xi \Xi^{T} \leq I$ or less if and only if there is $\lambda>0$ makes

$$
\mathbf{Y}_{1}+\lambda \mathbf{M} \mathbf{M}^{\mathrm{T}}+\lambda^{-1} \mathbf{N}^{\mathrm{T}} \mathbf{N}<0
$$

If true, the above equation can be written as

$$
\mathbf{Y}_{1}+\left[\lambda^{(1 / 2)} \mathbf{M} \lambda^{-(1 / 2)} \mathbf{N}^{T}\right]\left[\begin{array}{c}
\lambda^{(1 / 2)} \mathbf{M}^{T} \\
\lambda^{-(1 / 2)} \mathbf{N}
\end{array}\right]<0
$$

Using Schur complement lemma, the above equation can be rewritten as

$$
\left[\begin{array}{ccc}
Y_{1} & {\left[\lambda^{(1 / 2)} M\right.} & \left.\lambda^{-(1 / 2)} N^{T}\right] \\
* & -I
\end{array}\right]<0 .
$$

Finally, multiplying equation (45) on both sides by $\operatorname{diag}\left\{\mathbf{I}, \mathbf{I}, \lambda^{(1 / 2)} \mathbf{I}, \lambda^{(1 / 2)} \mathbf{I}\right\}$, we could obtain $\Upsilon<0$.

\section{Control Algorithm}

The control algorithm for the proposed method is described below in the form of step-by-step algorithm implementation process as shown in Figure 1 as a flow diagram.

Step 1. Obtain the model parameters of the controlled object and transform them into a discrete time-invariant system as shown in equation (1). If there is a solution, then go to the next process and adopt gain and learning laws applied. If 


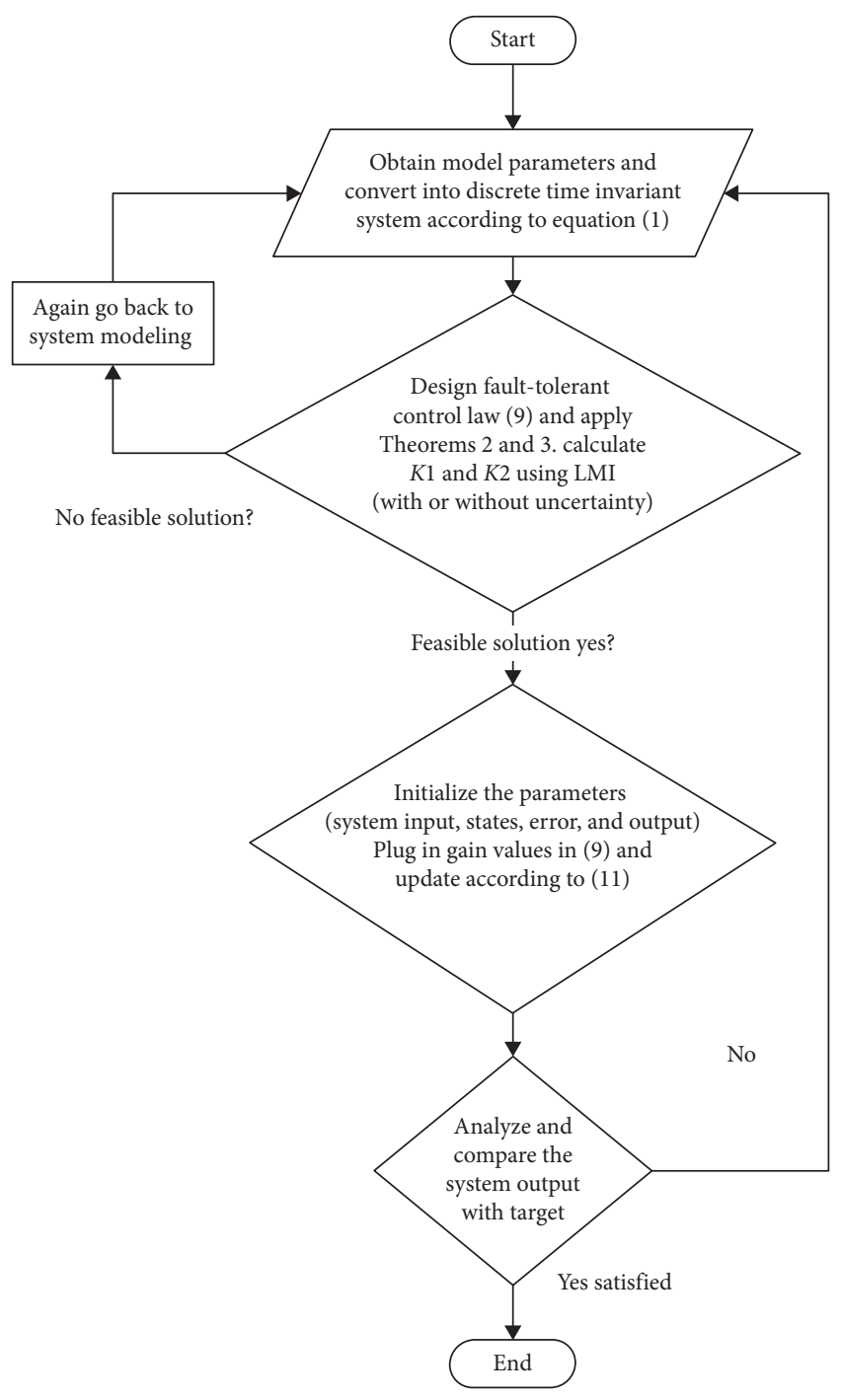

Figure 1: Control and design of algorithm step-by-step process.

there is no solution, then obviously, we need to calculate again system parameters accordingly.

Step 2. Design iterative learning fault-tolerant control law in the form of formula (11), and apply LMI toolbox in MATLAB to solve Theorems 2 or 3 according to the parameters given in formula (1), and obtain the parameters $K 1$ and $K 2$ of the system with and without uncertainty in formula (1), respectively. If there is a feasible solution, proceed to the next step; otherwise, redesign.

Step 3. According to the given reference target and system of the initial boundary conditions, the system of Equation (1) is taken after the initial iteration value, the state of the output and error when the initial iteration ended. And then, in Step 2 to solve the parameters $K_{1}$ and $K_{2}$ plug type (9) in the form of iterative learning control law, according to the type (11) and iterative learning control in the form of reconsideration, iterative learning controller is constantly modified.
Step 4. Observe the output response curve of the system, compare it with the expectation curve of a given reference target, and draw the root-mean-square (RMS) error curve. If the system output can track the expectation curve with a small error after several batches, the calculation method will be finished. Otherwise, if the tracking error is too large or the output response curve fails to track the expected curve after multiple batches, the controller shall be redesigned and Step 2 shall be returned.

\section{Practical Applications of the Proposed Controller}

6.1. Practical Simulation Example 1. To verify the validity of the method in this paper, the injection velocity control process of the reinjection molding process was taken as the research object [67]:

$$
\left\{\begin{array}{l}
x(t+1, k)=(A+\Delta A) x(t, k)+(B+\Delta B) u(t, k)+f(x(t, k)) \\
y(t, k)=(C+\Delta C) x(t, k)
\end{array}\right.
$$

Among them, system moment matrix is as follows:

$$
\begin{aligned}
& A=\left[\begin{array}{cc}
1.582 & -0.5916 \\
1 & 0
\end{array}\right], \\
& B=\left[\begin{array}{ll}
1 & 0
\end{array}\right]^{T}, \\
& C=\left[\begin{array}{ll}
1.69 & 1.419
\end{array}\right] .
\end{aligned}
$$

The expected trajectory is defined as

$$
y_{d}(t)= \begin{cases}\sin (0.01 \pi t), & 1 \leq t<200, \\ 1, & 200 \leq t<300, \\ 4-0.01 t, & 300 \leq t \leq 400 .\end{cases}
$$

At time $\mathrm{t} \in[0,400]$, the initial state of each batch is 0 . Suppose that the system's operating frequency norm $[0, \infty]$ is divided into $[0, \infty]=[0,6] \cup[6,25] \cup[25, \infty]$. After the 20th iteration, the occurrence actuator's fault suddenly appears, and the fault in the system is $f(t)=0.7+0.3 \times \sin (0.5 t)$. Evaluate performance and introduce performance indicators:

$$
\operatorname{RMS}(e(t, k))=\sqrt{\frac{1}{400} \sum_{t=1}^{400} e^{2}(t, k)} .
$$

Supposing that the coefficients of matrices $A$ and $B$ contain uncertainties, the decomposition of the uncertainty is carried out as follows: if the uncertainty matrix of the system $H 1=[-0.05,0.1], H 2=[-0.12,0.15], F 1=I, F 2=$ $[1,1]^{T}, \Xi=\operatorname{diag}\left\{\sigma_{1}, \sigma_{2}\right\}$, and $s 1$ and $\sigma 2$ are $-1 \sim 1$, then the gain matrix of the fault-tolerant controller is $K 1=[-1.5749$, 0.7293 ] and $K 2$ [0.1291].

Suppose the injection molding process is assumed to work in the intermediate frequency segment. In that case, the system RMS error's simulation curves and the number of overlaps are shown in Figures 2 and 3. By comparing the iterative fault-tolerant control results, it can be seen that the fault-tolerant control results designed within the specific domain of the system can accelerate the convergence speed 


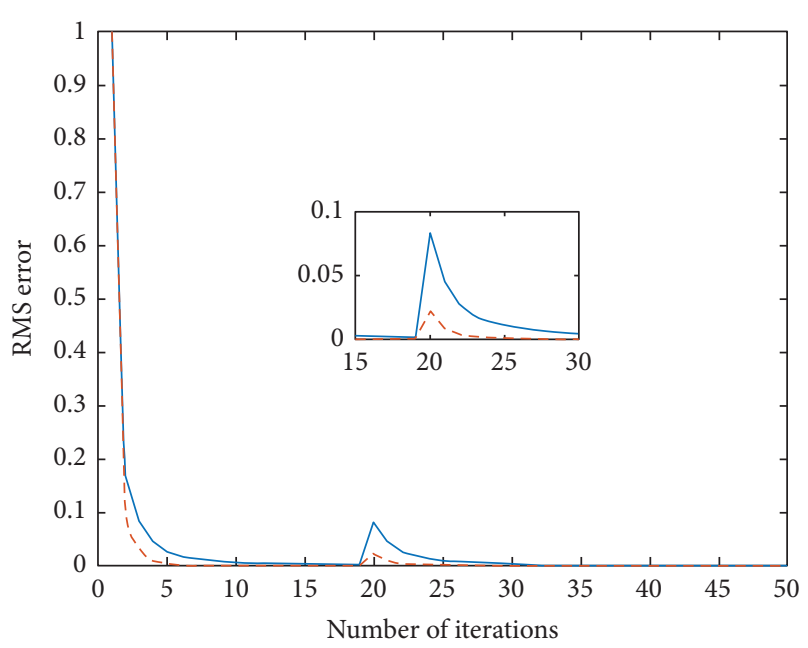

FIGURE 2: RMS error with and without uncertainty.

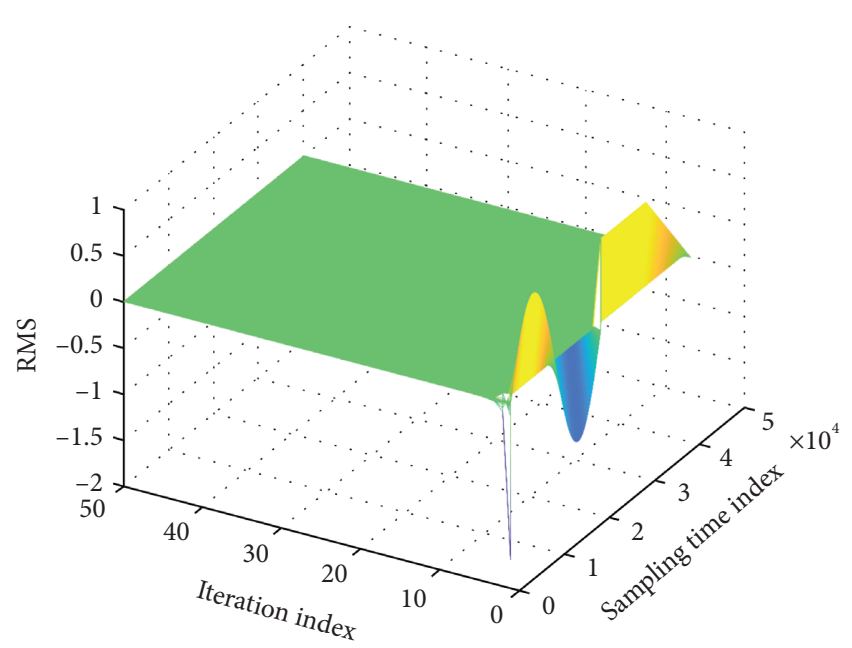

FIgURE 3: RMS error in time and iteration domain.

to some extent and can better restrain the influence of the operator's fault. The controller's uncertainty and fault-tolerant capability are quite robust, limiting the text and not be repeated. As shown in Figure 2, the system tracking error decreases quickly with the increase of iteration time before the actuator fails. After the actuator failed in iteration 20, the system's tracking performance decreased significantly. However, under the control of the fault-tolerant controller, with the increasing number of iterations, the system recovered quickly and steadily, indicating that the system had a good follow-up effect under normal and actuator failure conditions.

The simulation outcomes and effects of the control law are shown in Figures 2 and 3. Even with nonrepetitive disturbances, the tracking error of the first 20 batches of the system before the failure occurred quickly converged to a stable state with respect to time, and the tracking performance continued to improve in the batch direction, as shown in Figure 2, in the 25th batch of process failures. After it happened, the tracking performance of the system was lower than that of the 25th batch. However, after several batch iterations, the tracking performance reached an ideal level again, as shown in Figure 3. This conclusion is reflected in Figures 2 and 3. Figure 3 shows the daily values of different batches, and Figure 4 shows the tracking error of the system in a three-dimensional graph.

6.2. Practical Simulation Example 2. In order to verify the effectiveness of the algorithm proposed in this paper, the single-link manipulator that performs the motion trajectory tracking task repeatedly is considered as the experimental object. The continuous time model of the single-link manipulator system is described as [68]

$$
\tau(t)=M t^{2} \frac{\mathrm{d}^{2} \theta(t)}{\mathrm{d} t^{2}}+V \frac{\mathrm{d} \theta(t)}{\mathrm{d} t}+M_{g} l \cos (\theta(t)),
$$

where $\theta(t)$ is manipulator joints of angular displacement, $\tau(t)$ is the driving moment of joints, $l=0.6 \mathrm{~m}$ represents the length of the manipulator rod, $m=0.6 \mathrm{~kg}$ is the quality of the manipulator, $V=1.5\left(\mathrm{~kg} / \mathrm{m}^{2} / \mathrm{s}\right)$ is the mechanical arm joint friction resistance coefficient, and $g=9.8\left(\mathrm{~m} / \mathrm{s}^{2}\right)$ for the acceleration of gravity.

Through Euler's method, discrete sampling is carried out for the continuous system (34) at time interval $h=0.2 \mathrm{~s}$, and $T$ represents the discrete sampling point. At the same time, $x_{1}(t, k)=(h t, k)$ and $\quad x_{2}(t, k)=(h t+h, k), \quad$ respectively, represent the state variable of the $k$ batch of the repetitive operation of the single-link decentralization system, $u(t, k)=$ $(h T, k)$ as the control input, $y(T, k)=(h t+h, k)$ as the system output, and the discrete time-invariant nonlinear model of the single-link system (50) can be represented as

$$
\begin{aligned}
x_{1}(t+1, k)= & x_{2}(t, k) x_{2}(t+1, k) \\
= & \left(2-\frac{v h}{M t^{2}}\right) x_{2}(t, k)+\left(\frac{v h}{M l^{2}}-1\right) x_{1}(t, k) \\
& -\frac{g h^{2}}{l} \cos \left(x_{1}(t, k)\right)+\frac{h^{2}}{M l^{2}} .
\end{aligned}
$$

A discrete time-invariant nonlinear model consistent with the form of equation (1) can be further obtained:

$$
\begin{aligned}
& A=\left[\begin{array}{cc}
0 & 0 \\
\frac{V h}{M l^{2}}-1 & 2-\frac{V h}{M l^{2}}
\end{array}\right]=\left[\begin{array}{cc}
0 & 1 \\
0.39 & 0.61
\end{array}\right], \\
& B=\left[\begin{array}{c}
0 \\
\frac{h^{2}}{M l^{2}}
\end{array}\right]=\left[\begin{array}{c}
0 \\
0.19
\end{array}\right], \\
& C=\left[\begin{array}{ll}
0 & 1
\end{array}\right] .
\end{aligned}
$$

The nonlinear function is 


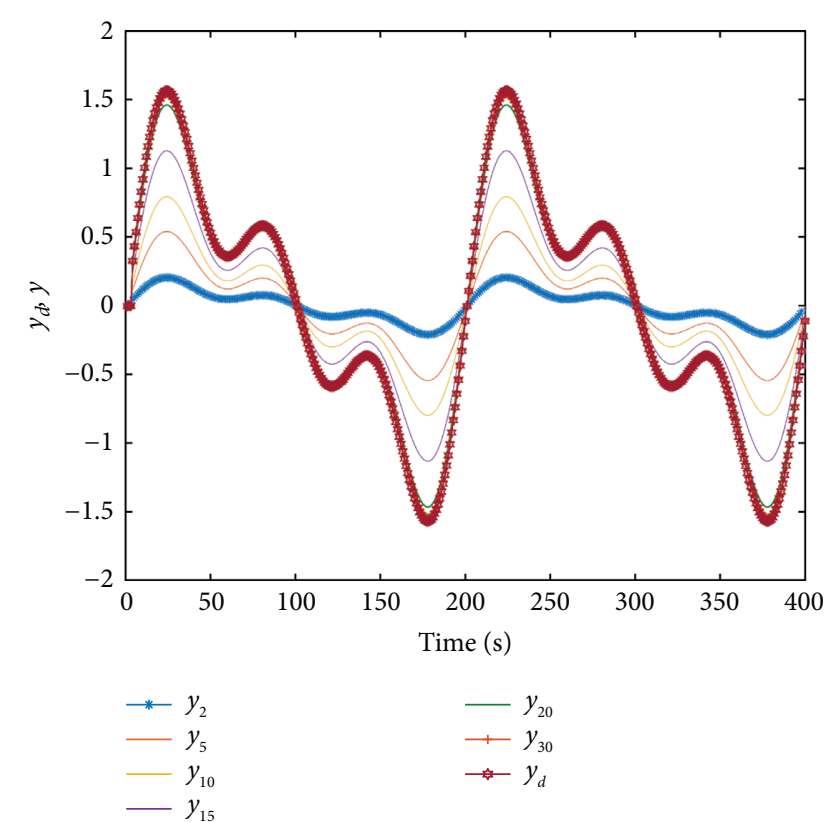

FIGURE 4: Output versus reference trajectory curve without actuator failure.

$$
f(x(t, k))=\left[\begin{array}{c}
0 \\
-\frac{g h^{2}}{l}
\end{array}\right] \cos \left(x_{1}(t, k)\right)=\left[\begin{array}{c}
0 \\
0.653
\end{array}\right] \cos \left(x_{1}(t, k)\right) .
$$

Because $\cos \left(x_{1}(t, k)\right)$ has upper and lower bounds, so for nonlinear function, $f(x(t, k))=\left[\begin{array}{c}0 \\ 0.653\end{array}\right] \cos \left(x_{1}(t, k)\right)$ satisfies the nonlinear constraint (2). At the same time, it is assumed that the manipulator needs to run 100 batches repeatedly and track the reference trajectory:

$$
y_{d}(t)=\sin \left(\frac{\pi t}{100}\right)+0.5 \sin \left(\frac{\pi t}{200}\right)+0.5 \sin \left(\frac{\pi t}{300}\right) .
$$

Initially, we can assume that our system's initial condition is zero like input $u(t, k)=(0,0)$ states $x_{1}(t, k)=(0,0)$ and $x_{2}(t, k)=(0,0)$ and output $y(T, k)=(0,0) 0$, respectively. Due to the repeated operation of the industrial application of mechanical arm often, we assume that the 50th connecting rod will be shown after the repeat problem such as mechanical wear. The failure occurs after each batch of the intermittent fault happens in the process, and the failure changes in each iteration with respect to time. And the manipulator joints' input driving moment will change with fault time-varying characteristics. The typical case of this fault is the gear transmission part of the manipulator; after a long period of operation, there will be missing teeth, broken teeth, and other phenomena, resulting in the system in each operating batch of the cycle time time-varying failure. Therefore, it is assumed that the system input is an actuator fault in the form of equation (3), and the fault coefficient $=(0.15+0.85 \sin (0.5 t))$.
Assuming that there is no uncertainty, the gain $K 1=[-1.873$ $2-4.1186$ ] and $K 2=0.5673$ of the modified update quantity (13) in the iterative learning fault-tolerant controller (11) is solved according to Theorem 2 and LMI tool.

In addition, it is noted that, in the manipulator system in actual operation, the joint frictional resistance coefficient $\mathrm{V}$, rod length $L$, and mass $M$ in matrices $\mathrm{A}$ and $B$ will become uncertain due to wear and other factors, so the variation of these uncertainty factors should be considered in the controller design process. Assuming that the coefficients of matrices $\mathrm{A}$ and $\mathrm{B}$ contain uncertainties, the uncertainty decomposition is performed as follows:

$$
\begin{aligned}
\mathbf{H}_{1} & =\left[\begin{array}{cc}
0 & 0 \\
-0.05 & 0.1
\end{array}\right], \\
\mathbf{H}_{2} & =\left[\begin{array}{ll}
0 & 0
\end{array}\right], \\
F_{1} & =I, \\
F_{2} & =\left[\begin{array}{ll}
0 & 1
\end{array}\right] T,
\end{aligned}
$$

where $\Xi=\operatorname{diag}\left\{\sigma_{1}, \sigma_{2}\right\}, \sigma 1$ and $\sigma 2$ are -1 to 1 in between numerical values. $\Delta A=H_{1} \Xi F_{1}=\left[\begin{array}{cc}0 & 0 \\ -0.05 \sigma 1 & 0.1 \sigma 2\end{array}\right]$, $\Delta B=H_{1} \Xi F_{2}=\left[\begin{array}{c}0 \\ 0.1 \sigma 2\end{array}\right]$, and $\Delta C=H_{2} \Xi F_{2}=0$.

At this point, according to the conclusion of Theorem 3, we know that the system of the gain of modified update quantity (13) in iterative learning robust fault-tolerant controller (11) can be obtained by using LMI tool, $K_{1}=$ $\left[\begin{array}{ll}-2.4149 & -4.6484\end{array}\right]$ and $K_{2}=1.9840$.

In order to evaluate the control effect of each batch of manipulator during repeated operation, the RMS value of tracking error $E(t, k)$ was defined as the tracking energy index

$$
\operatorname{RMS}(e(t, k))=\sqrt{\frac{1}{400} \sum_{i=1}^{400} e^{2}(t, k)}
$$

In the formula, the smaller RMS $(\mathrm{E}(t, \mathrm{k}))$ is, the better the tracking effect of lot $\mathrm{K}$ is. The tracking control simulation results of the single-link manipulator system under the nondeterministic conditions are shown in Figures 4-6, respectively.

It can be clearly observed that the output of the system is trying to track the reference tracking trajectory of the system as shown in Figure 4. As can be seen from Figure 5, the tracking error of the system decreases rapidly with the increase of iteration times before the actuator fails. After the failure of the actuator in batch 50, the tracking performance of the system decreased significantly. However, under the action of the fault-tolerant controller, with the increasing number of iterations, the system collected again rapidly and stably, indicating that the system achieved a good tracking effect under both normal and failure conditions of the actuator. Figure 4 shows the system output curve and 


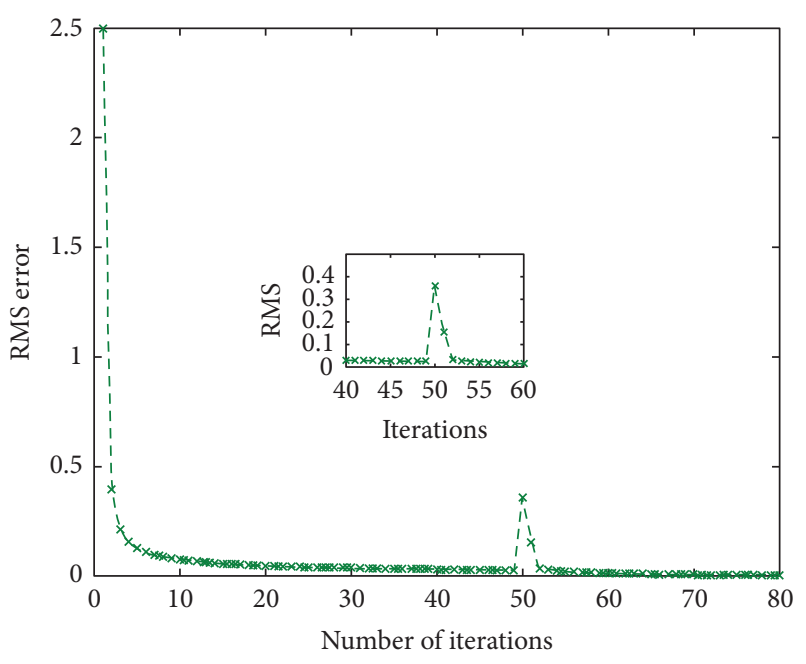

FIGURE 5: RMS error curve without actuator failure.

expectation curve before the actuator failure, and the threedimensional figure of Figure 6 represents the tracking error change of the system in two dimensions of the batch axis and time axis. It can be seen that, with the increase of the number of iterations, the actual output curve of the manipulator can gradually track the upward periodic curve. Correspondingly, the simulation results of robust faulttolerant control when the system is uncertain are shown in Figures 7-9, respectively.

The simulation results are shown in Figures 4 and 5 . Even if the disturbance is repeated, the tracking error of the first 50 iterations of the system before the fault occurs rapidly converges to a stable state with time, and the tracking performance keeps improving in the direction of the batches, as shown in Figure 7. After the failure of the first batch, the tracking performance of the system decreased compared with that of the 50th batch. However, after several batch iterations, the tracking performance reached an ideal level again, as shown in Figure 8. This conclusion is shown in Figures 8 and 9, which show the regular values of different batches of iterations, and Figure 9, which shows the tracking error of the system in 3D. It can be observed that under the effect of time-varying failure and repetitive disturbance, and actuator failure, the proposed algorithm in the normal situation and therefore failure can guarantee system in both iteration and time direction monotone convergent. According to the asymptotic stability, a designed controller has fault tolerance, and fault tolerance of both performed at the same time and has a robust inhibitory effect on nonrecurring interference.

Figures 4-9 shows that, under the use of time-varying faults and nonrepetitive disturbances, the proposed algorithm can guarantee monotonic convergence of the system in both batch and time directions under normal and fault scenarios, and it is asymptotically stable. The designed controller has both fault-tolerant and fault-tolerant performances and has a robust suppression effect on nonrepetitive external interference.

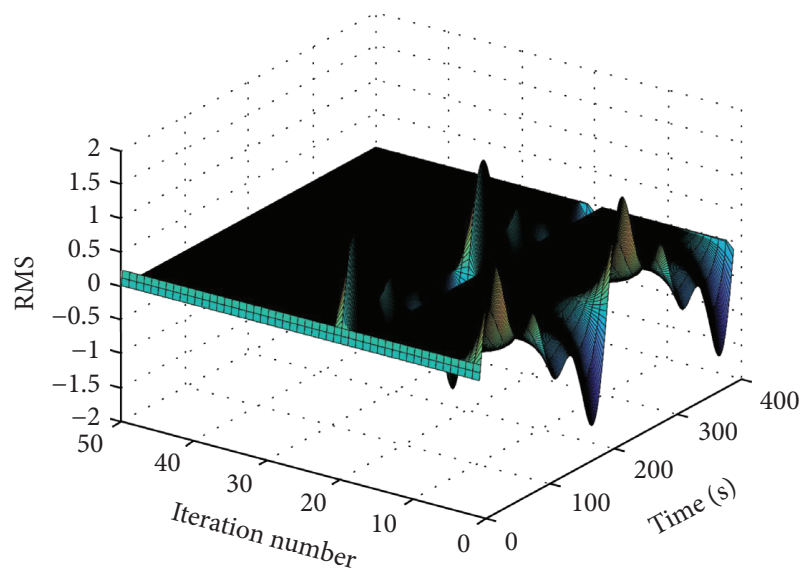

FIgURE 6: Error trajectory in both time and iteration domain (without actuator failure).

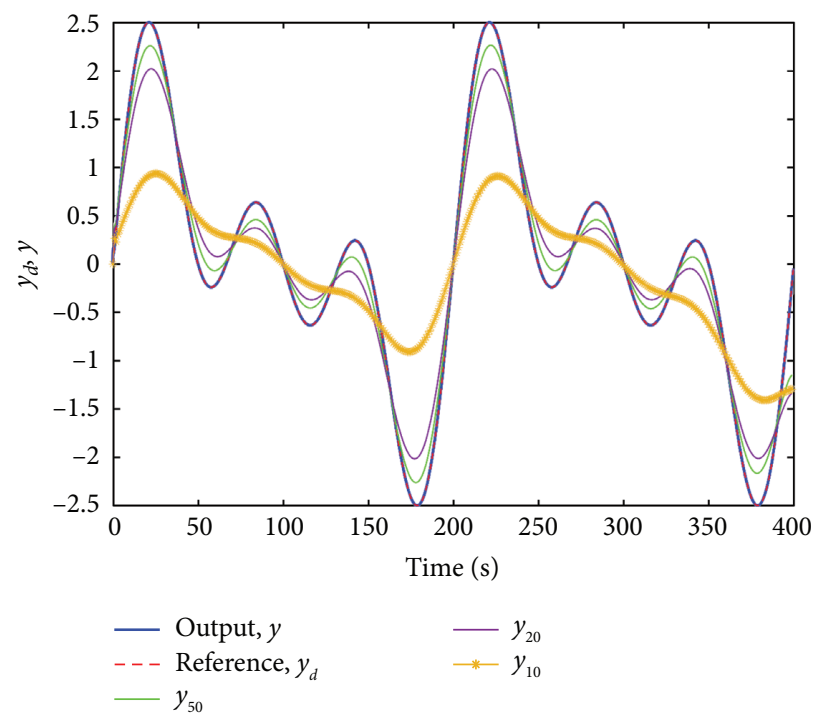

FIgURE 7: Output versus reference trajectory curve with actuator failure.

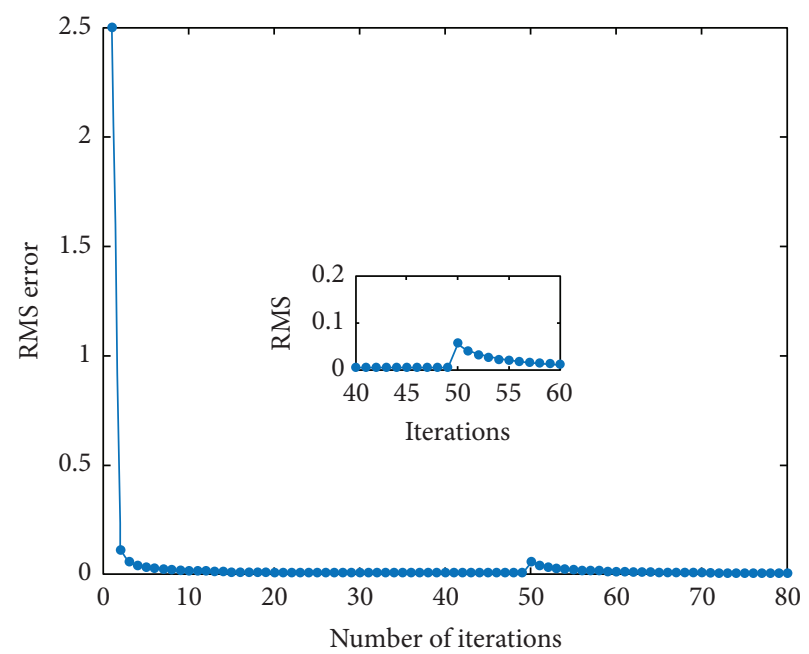

FIGURE 8: RMS error curve with actuator failure. 


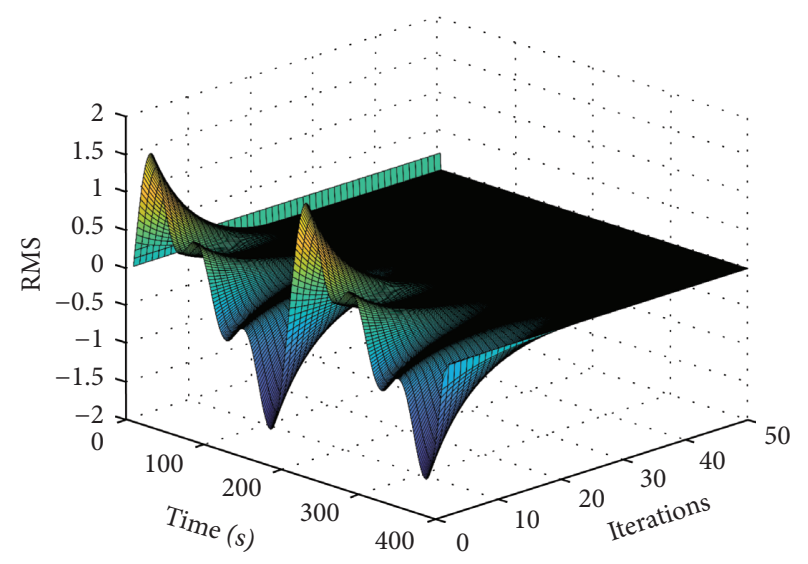

FIGURE 9: Error trajectory in both time and iteration domain (with actuator failure).

\section{Conclusion}

The iterative learning fault-tolerant control algorithm for a class of uncertain discrete time-invariant nonlinear systems with actuator faults is studied in this paper. First, the sufficient conditions for the stability of the nonlinear discrete repetition process are obtained by the Lyapunov method, the gain of the iterative learning fault-tolerant controller is solved by LMI, and the robustness of the system with uncertainty is discussed. Finally, the feasibility of the proposed algorithm is verified by the simulation experiment of the single-link manipulator as well as the electromechanical system for batch process. The method proposed in this paper is a further extension of the iterative learning control method for linear repeating process, which is obviously more adaptable and effective for the repeated operation process with nonlinear links in the industrial process.

The proposed algorithm improves the error identification and estimation of nonlinearly unstable systems, enhances error detection reliability, and reduces false alarms. The faults of nonlinear models based on iterative learning are more up-front and more suitable for technical applications. Based on the method proposed in this paper, we can also use it to identify and estimate uncertain nonlinear systems. The future applications of this proposed algorithm are as follows:

(i) Aircraft actuator fault-tolerant control system

(ii) Robotics (soft robotics, elastic actuators, and omniwheel robots) trajectory control

(iii) Robustification in PMSM motor servo applications

(iv) Nonlinear servo speed and position control system

(v) Piezoelectric position and force tracking controls

\section{Data Availability}

The data are available and can be provided upon request. The simulation examples are taken from two sources: (1) J. Shi, F. Gao, and T. J. Wu, "Robust design of integrated feedback and iterative learning control of a batch process based on a 2D Roesser system," Journal of Process Control, vol. 15, no. 8, pp. 907-924, 2005; (2) D.-H. Hwang, Z. Bien, and S.-R.
Oh, "Iterative learning control method for discrete-time dynamic systems," in IEE Proceedings D-Control Theory and Applications, 1991, vol. 138, no. 2, pp. 139-144: IET.

\section{Conflicts of Interest}

The authors declare that they have no conflicts of interest.

\section{References}

[1] M. Hamidaoui, C. Shao, and S. Haouassi, "A PD-type iterative learning control algorithm for one-dimension linear wave equation," International Journal of Control, Automation and Systems, vol. 18, no. 4, pp. 1045-1052, 2020.

[2] L. Zhou, L. Jia, Y.-L. Wang, D. Peng, and W. Tan, "An integrated robust iterative learning control strategy for batch processes based on 2D system," Journal of Process Control, vol. 85, pp. 136-148, 2020.

[3] S. Riaz, H. Lin, M. Bilal Anwar, and H. Ali, "Design of PD-type second-order ILC law for PMSM servo position control," Journal of Physics: Conference Series, vol. 1707, Article ID 012002, 2020.

[4] Z. Li, Y. Hu, and D. Li, "Robust design of feedback feed-forward iterative learning control based on 2D system theory for linear uncertain systems," International Journal of Systems Science, vol. 47, no. 11, pp. 2620-2631, 2016.

[5] S. Riaz, H. Lin, and H. Elahi, "A novel fast error convergence approach for an optimal iterative learning controller," Integrated Ferroelectrics, vol. 213, no. 1, pp. 103-115, 2020.

[6] S. Arimoto, S. Kawamura, and F. Miyazaki, "Bettering operation of robots by learning," Journal of Robotic Systems, vol. 1, no. 2, pp. 123-140, 1984.

[7] Y. Wang, J. Shi, D. Zhou, and F. Gao, "Iterative learning fault-tolerant control for batch processes," Industrial \& Engineering Chemistry Research, vol. 45, no. 26, pp. 9050-9060, 2006.

[8] B. Pang and C. Shao, "PID-type parameter optimal iterative learning control algorithm based on singular value decomposition [J]," Information and Control, vol. 43, no. 4, pp. 483-489, 2014.

[9] S. Riaz, H. Lin, and M. P. Akhter, "Design and implementation of an accelerated error convergence criterion for norm optimal iterative learning controller," Electronics, vol. 9, no. 11, p. 1766, 2020.

[10] S.-L. Xie, S.-P. Tian, and Z. Xie, Iterative Learning Control Theory and Application, Science Press, Beijing, China, 2005.

[11] C. Freeman and Y. Tan, Iterative Learning Control and Repetitive Control, Taylor \& Francis, Oxfordshire, UK, 2011.

[12] C. T. Freeman, Z. Cai, E. Rogers, and P. L. Lewin, "Iterative learning control for multiple point-to-point tracking application," IEEE Transactions on Control Systems Technology, vol. 19, no. 3, pp. 590-600, 2010.

[13] Z. Gao, X. Liu, and M. Z. Chen, "Unknown input observerbased robust fault estimation for systems corrupted by partially decoupled disturbances," IEEE Transactions on Industrial Electronics, vol. 63, no. 4, pp. 2537-2547, 2015.

[14] D. Rotondo, F. Nejjari, V. Puig, and J. Blesa, "Model reference FTC for LPV systems using virtual actuators and set-membership fault estimation," International Journal of Robust and Nonlinear Control, vol. 25, no. 5, pp. 735-760, 2015.

[15] B. Zhao, D. Liu, and Y. Li, "Observer based adaptive dynamic programming for fault tolerant control of a class of nonlinear systems," Information Sciences, vol. 384, pp. 21-33, 2017. 
[16] L. Liu, Z. Wang, and H. Zhang, "Adaptive fault-tolerant tracking control for MIMO discrete-time systems via reinforcement learning algorithm with less learning parameters," IEEE Transactions on Automation Science and Engineering, vol. 14, no. 1, pp. 299-313, 2016.

[17] X. Li, D. Lu, G. Zeng, J. Liu, and W. Zhang, "Integrated fault estimation and non-fragile fault-tolerant control design for uncertain Takagi-Sugeno fuzzy systems with actuator fault and sensor fault," IET Control Theory \& Applications, vol. 11, no. 10, pp. 1542-1553, 2017.

[18] J. A. Guzmán-Rabasa, F. R. López-Estrada, B. M. GonzálezContreras, G. Valencia-Palomo, M. Chadli, and M. Perez-Patricio, "Actuator fault detection and isolation on a quadrotor unmanned aerial vehicle modeled as a linear parameter-varying system," Measurement and Control, vol. 52, no. 9-10, pp. 1228-1239, 2019.

[19] X. Nian, W. Chen, X. Chu, and Z. Xu, "Robust adaptive fault estimation and fault tolerant control for quadrotor attitude systems," International Journal of Control, vol. 93, no. 3, pp. 725-737, 2020.

[20] B. Luo, Y. Yang, and D. Liu, “Adaptive \$Q\$ -learning for data-based optimal output regulation with experience replay," IEEE Transactions on Cybernetics, vol. 48, no. 12, pp. 3337-3348, 2018.

[21] M. Schmid, E. Gebauer, C. Hanzl, and C. Endisch, "Active model-based fault diagnosis in reconfigurable battery systems," IEEE Transactions on Power Electronics, vol. 36, no. 3, pp. 2584-2597, 2020.

[22] C. Edwards and C. P. Tan, "Sensor fault tolerant control using sliding mode observers," Control Engineering Practice, vol. 14, no. 8, pp. 897-908, 2006.

[23] S. Du and S. Razavi, "Fault-tolerant control of variable speed limits for freeway work zone using likelihood estimation," Advanced Engineering Informatics, vol. 45, p. 101133, 2020.

[24] G. Xiao and F. Liu, "Observer-based cooperative distributed fault-tolerant model predictive control with imperfect network communication and asynchronous measurements," International Journal of Robust and Nonlinear Control, vol. 30, no. 12, pp. 4531-4549, 2020.

[25] X. Feng and Y. Wang, "Fault estimation based on sliding mode observer for Takagi-Sugeno fuzzy systems with digital communication constraints," Journal of the Franklin Institute, vol. 357, no. 1, pp. 569-588, 2020.

[26] G. Li, S. J. Qin, Y. Ji, and D. Zhou, "Reconstruction based fault prognosis for continuous processes," Control Engineering Practice, vol. 18, no. 10, pp. 1211-1219, 2010.

[27] S. Riaz, H. Elahi, K. Javaid, and T. Shahzad, "Vibration feature extraction and analysis for fault diagnosis of rotating machinery-a literature survey," Asia Pacific Journal of Multidisciplinary Research, vol. 5, 2017.

[28] R. Arunthavanathan, F. Khan, S. Ahmed, S. Imtiaz, and R. Rusli, "Fault detection and diagnosis in process system using artificial intelligence-based cognitive technique," Computers \& Chemical Engineering, vol. 134, Article ID 106697, 2020.

[29] Y. Zhao, T. Li, X. Zhang, and C. Zhang, "Artificial intelligencebased fault detection and diagnosis methods for building energy systems: advantages, challenges and the future," Renewable and Sustainable Energy Reviews, vol. 109, pp. 85-101, 2019.

[30] B. Jiang and M. Staroswiecki, "Adaptive observer design for robust fault estimation," International Journal of Systems Science, vol. 33, no. 9, pp. 767-775, 2002.

[31] S. He and F. Liu, "Adaptive observer-based fault estimation for stochastic Markovian jumping systems," Abstract and Applied Analysis, vol. 2012, Article ID 176419, 11 pages, 2012.
[32] H. Li, F. You, and F. Wang, "A delay dependent approach to robust fast adaptive fault estimation design for uncertain neutral systems with time delay," Transactions of the Institute of Measurement and Control, vol. 40, no. 8, pp. 2579-2588, 2018.

[33] M. Zhou, Z. Wang, and Y. Shen, "Simultaneous fault estimation and fault-tolerant tracking control for uncertain nonlinear discrete-time systems," International Journal of Systems Science, vol. 48, no. 7, pp. 1367-1379, 2017.

[34] J. Sahng, M.-Y. Chen, and D.-H. Zhou, "Incipient fault detection using transformed component statistical analysis," Journal of Shanghai Jiaotong University, vol. 6, p. 11, 2015.

[35] Ã. bǑè, “¿ íûä Đ î åHo Ho," Acta Automatica Sinica, vol. 36, no. $12,2010$.

[36] L. Wang, H. Pan, J. Gao, and D. Chen, "Predictive compensation for wireless networked system with time delay and packet dropout based on TS model," in Abstract and Applied Analysis, vol. 2014, Article ID 953039, 8 pages, 2014.

[37] S. Riaz, H. Lin, and H. Elahi, "A novel fast error convergence approach for an optimal iterative learning controller," Integrated Ferroelectrics, vol. 213, 2021.

[38] J. Han, H. Zhang, Y. Wang, and K. Zhang, "Fault estimation and fault-tolerant control for switched fuzzy stochastic systems," IEEE Transactions on Fuzzy Systems, vol. 26, no. 5, pp. 2993-3003, 2018.

[39] F. Li, Z. Hu, and G. Zhao, "Fault estimation and adaptive fault tolerant control for dynamic systems based on the second-order sliding mode observer," Proceedings of the Institution of Mechanical Engineers, Part I: Journal of Systems and Control Engineering, vol. 230, no. 3, pp. 222-230, 2016.

[40] Z. Hu, G. Zhao, L. Zhang, and D. Zhou, "Fault estimation for nonlinear dynamic system based on the second-order sliding mode observer," Circuits, Systems, and Signal Processing, vol. 35, no. 1, pp. 101-115, 2016.

[41] B. Jiang, V. Cocquempot, and M. Staroswiecki, "Fault estimation in nonlinear uncertain systems using robust/ sliding-mode observers," IEE Proceedings-Control Theory and Applications, vol. 151, no. 1, pp. 29-37, 2004.

[42] F. Haouari, M. Djemai, and B. Cherki, "Sliding mode observers for TS fuzzy systems with application to sensor fault estimation," in Proceedings of the 2015 3rd International Conference on Control, Engineering \& Information Technology (CEIT), pp. 1-5, IEEE, Tlemcen, Algeria, May 2015.

[43] Z. Ming-Yue, L. He-Ping, L. Zhi-Jun, S. De-Hui, and L. KePing, "Fault tolerant control for networked control systems with access constraints," Acta Automatica Sinica, vol. 38, no. 7, pp. 1119-1126, 2012.

[44] K. Zhao, P. Li, C. Zhang, X. Li, J. He, and Y. Lin, "Sliding mode observer-based current sensor fault reconstruction and unknown load disturbance estimation for PMSM driven system," Sensors, vol. 17, no. 12, p. 2833, 2017.

[45] J. Mackerle, "Finite element modelling of ceramics and glass, an addendum - a bibliography (1998-2004)," Engineering Computations, vol. 22, no. 3, 2005.

[46] L. Wang, F. Liu, J. Yu, P. Li, R. Zhang, and F. Gao, "Iterative learning fault-tolerant control for injection molding processes against actuator faults," Journal of Process Control, vol. 59, pp. 59-72, 2017.

[47] D. Rotondo, F.-R. López-Estrada, F. Nejjari, J.-C. Ponsart, D. Theilliol, and V. Puig, "Actuator multiplicative fault estimation in discrete-time LPV systems using switched observers," Journal of the Franklin Institute, vol. 353, no. 13, pp. 3176-3191, 2016. 
[48] D. Du, S. Xu, and V. Cocquempot, "Actuator fault estimation for discrete-time switched systems with finite-frequency," Systems \& Control Letters, vol. 108, pp. 64-70, 2017.

[49] H. Elahi, "The investigation on structural health monitoring of aerospace structures via piezoelectric aeroelastic energy harvesting," Microsystem Technologies, pp. 1-9, 2020.

[50] H. Elahi, M. Eugeni, F. Fune et al., "Performance evaluation of a piezoelectric energy harvester based on flag-flutter," Micromachines, vol. 11, no. 10, p. 933, 2020.

[51] S. Hara, Y. Yamamoto, T. Omata, and M. Nakano, "Repetitive control system: a new type servo system for periodic exogenous signals," IEEE Transactions on Automatic Control, vol. 33, no. 7, pp. 659-668, 1988.

[52] M. IKEDA and M. TAKANO, "Repetitive control for systems with nonzero relative degree," Transactions of the Society of Instrument and Control Engineers, vol. 24, no. 6, pp. 575-582, 1988.

[53] H. Elahi, K. Munir, M. Eugeni, S. Atek, and P. Gaudenzi, "Energy harvesting towards self-powered IoT devices," Energies, vol. 13, no. 21, p. 5528, 2020.

[54] Y. Zhang and J. Jiang, "Issues on integration of fault diagnosis and reconfigurable control in active fault-tolerant control systems," IFAC Proceedings Volumes, vol. 39, no. 13, pp. 1437-1448, 2006.

[55] W. Limin, C. Xi, and G. Furong, "An LMI method to robust iterative learning fault-tolerant guaranteed cost control for batch processes," Chinese Journal of Chemical Engineering, vol. 21, no. 4, pp. 401-411, 2013.

[56] L. Wang, S. Mo, D. Zhou, F. Gao, and X. Chen, "Robust delay dependent iterative learning fault-tolerant control for batch processes with state delay and actuator failures," Journal of Process Control, vol. 22, no. 7, pp. 1273-1286, 2012.

[57] J. Wang, Y. Li, G. Hu, and M. Yang, "Lightweight research in engineering: a review," Applied Sciences, vol. 9, no. 24, p. 5322, 2019.

[58] X. Bu, F. Yu, Z. Hou, and H. Yang, "Robust iterative learning control for nonlinear systems with measurement disturbances," Journal of Systems Engineering and Electronics, vol. 23, no. 6, pp. 906-913, 2012.

[59] B. Cichy, K. Gałkowski, and E. Rogers, "2D systems based robust iterative learning control using noncausal finite-time interval data," Systems \& Control Letters, vol. 64, pp. 36-42, 2014.

[60] L. Wang, S. Mo, H. Qu, D. Zhou, and F. Gao, "Ho design of 2D controller for batch processes with uncertainties and interval time-varying delays," Control Engineering Practice, vol. 21, no. 10, pp. 1321-1333, 2013.

[61] M. French and E. Rogers, "Non-linear iterative learning by an adaptive Lyapunov technique," International Journal of Control, vol. 73, no. 10, pp. 840-850, 2000.

[62] M. Boski and W. Paszke, "Design of robust iterative learning control schemes for systems with polytopic uncertainties and sector-bounded nonlinearities," Journal of Physics: Conference Series, vol. 783, no. 1, p. 012032, 2017.

[63] P. V. Pakshin, J. P. Emelianova, M. A. Emelianov, K. Gałkowski, and E. Rogers, "Stochastic stability of some classes of nonlinear 2D systems," Automation and Remote Control, vol. 79, no. 1, pp. 89-102, 2018.

[64] S. Boyd, Linear Matrix Inequalities in System and Control Theory, Siam, Philadelphia, PA, USA, 1994.

[65] L. Xie, "Output feedback Ho control of systems with parameter uncertainty," International Journal of Control, vol. 63, no. 4, pp. 741-750, 1996.
[66] P. Gahinet and P. Apkarian, "A linear matrix inequality approach to Ho control," International Journal of Robust \& Nonlinear Control, vol. 4, pp. 421-448, 2010.

[67] J. Shi, F. Gao, and T.-J. Wu, "Robust design of integrated feedback and iterative learning control of a batch process based on a 2D Roesser system," Journal of Process Control, vol. 15, no. 8, pp. 907-924, 2005.

[68] D.-H. Hwang, Z. Bien, and S.-R. Oh, "Iterative learning control method for discrete-time dynamic systems," in IEE Proceedings D Control Theory and Applicationsvol. 138, no. 2, , pp. 139-144, IET, 1991. 